\title{
Missed Opportunities for Sedation and Pain Management at a Level III Neonatal Intensive Care Unit, India
}

\author{
Shikha Y. Kothari' , Ashish R. Dongara ${ }^{1}$, Somashekhar M. Nimbalkar ${ }^{1,2 *}$, \\ Ajay G. Phatak ${ }^{2}$ and Archana S. Nimbalkar ${ }^{3}$
}

${ }^{1}$ Department of Pediatrics, Pramukhswami Medical College, Karamsad-Anand, India, ${ }^{2}$ Central Research Services, Charutar Arogya Mandal, Karamsad-Anand, India, ${ }^{3}$ Department of Physiology, Pramukhswami Medical College, Karamsad-Anand, India

Background: Neonates in the neonatal intensive care unit (NICU) undergo a multitude of painful and stressful procedures during the first days of life. Stress from this pain can lead to neurodevelopmental problems that manifest in later childhood and should be prevented.

Objective: To determine the number of painful procedures performed per day for each neonate, to verify documentation of painful procedures performed, and to, subsequently, note missed opportunities for providing pain relief to neonates.

OPEN ACCESS

Edited by:

Hitesh Singh Sandhu, University of Tennessee Health

Science Center, USA

Reviewed by:

Vijay Srinivasan,

Children's Hospital of

Philadelphia, USA

Jesús López-Herce,

Hospital General Universitario

Gregorio Marañón, Spain

${ }^{*}$ Correspondence:

Somashekhar M. Nimbalkar somu_somu@yahoo.com

Specialty section: This article was submitted to Pediatric Critical Care,

a section of the journal

Frontiers in Pediatrics

Received: 30 November 2015 Accepted: 01 February 2016 Published: 23 February 2016

Citation: Kothari SY, Dongara AR, Nimbalkar SM, Phatak AG and Nimbalkar AS (2016) Missed Opportunities for Sedation and Pain Management at a Level III Neonatal Intensive Care Unit, India. Front. Pediatr. 4:7.

doi: 10.3389/fped.2016.00007
Methods: We conducted a cross-sectional study at a level III NICU located in a rural part of western India. A total of 69 neonates admitted for more than $24 \mathrm{~h}$ were included. Twenty-nine neonates were directly observed for a total of $24 \mathrm{~h}$ each, and another 40 neonatal records were retrospectively reviewed for the neonate's first 7 days of admission. All stressful and painful procedures performed on the neonate were recorded. Also recorded were any pharmaceutical pain relief agents or central nervous system depressants administered to the neonate before or at the time of the procedures. Average nurse-patient ratio was also calculated. Data were analyzed using descriptive statistics.

Results: A documentation deficit of $2.2 \%$ was observed. The average nurse-patient ratio was 1.53:1. A total of 13711 procedures were recorded, yielding 44.1 (38.1 stressful, 3.8 mildly painful, and 2.2 moderately painful) procedures per patient day. Common stressful procedures were position changing (2501) and temperature recording (2208). Common mildly and moderately painful procedures were heel prick (757) and endotracheal suctioning (526), respectively. Use of pharmacological agents coincided with $33.48 \%$ of the procedures. The choice of drug and time of administration were inappropriate, indicating that the pharmacological agents were intended not for pain relief but rather for a coexisting pathology or as sedation from ventilation with no analgesia.

Conclusion: Stressful procedures are common in the NICU; mildly and moderately painful procedures fairly common. Almost two-thirds of the times, no pharmaceutical pain relief methods were used, and when administered, the pharmaceutical agents were seldom intended for pain relief; this implies poor pain management practices and emphasizes the imperative need for educating NICU nurses, residents, fellows, and attendings.

Keywords: neonatal pain, opportunities, nurse, neonatal intensive care, critical care 


\section{INTRODUCTION}

The International Association for the Study of Pain (IASP) defines pain as "an unpleasant sensory and emotional experience associated with actual or potential tissue damage, or described in terms of such damage" (1). This definition is not applicable for certain population groups like newborns (2). Pain in newborns is a highly complex phenomenon that needs to be well understood by health-care personnel involved in neonatal care $(3,4)$.

The past two decades have seen an increase in knowledge regarding the physiology of neonatal pain, the development of multiple valid pain assessment tools, and the formulation of various guidelines for pain management in newborns. However, discrepancies and shortcomings in neonatal pain assessment and management still exist among various neonatal care units (5-7).

Neonates repeatedly exposed to pain tend to perceive pain as more severe (8). Such pain can lead to an alteration in the neonate's nociceptive circuitry in adulthood (9). There can be long-term neurodevelopmental, emotional abnormalities, and social-functional abnormalities (10). This alteration depends on the type and intensity of pain experienced (11). Preterm neonates have a lower threshold for pain and are therefore more affected by pain $(12,13)$. These factors need to be taken into consideration when neonatologists and neonatal intensive care unit (NICU) staff establish a management plan.

Painful and stressful procedures are an inevitable side effect of clinical management. A majority of neonates in the NICU undergo a multitude of painful and stressful procedures during the first few days of life $(14,15)$. Epidemiological studies have demonstrated that the pain induced by these procedures is neither identified properly nor adequately treated (16).

Past studies have demonstrated the importance of developing established, evidence-based prevention and treatment protocols for neonatal pain (17-19) and the benefits of incorporating pain management as a routine component of care provided to "all neonates, regardless of their gestational age or severity of illness" (17). In 2006, the American Academy of Pediatrics and the Canadian Pediatric Society collaborated to formulate guidelines recommending implementation of a "pain-prevention program" in every health-care facility providing care to neonates. According to these guidelines, such a program should include "strategies for routinely assessing pain, minimizing the number of painful procedures performed, effectively using pharmacologic and nonpharmacologic therapies for the prevention of pain associated with routine minor procedures, and eliminating pain associated with surgery and other major procedures" (20).

However, while such guidelines and recommendations have been in existence for nearly a decade in developed countries, neonatal pain and its prevention and management is still in its evolving phase in developing nations. Though National Neonatology Forum (NNF) of India established guidelines regarding neonatal procedural pain and its management a few years ago (13), there is insufficient data available regarding the frequency of procedural exposure, the identification and/or the prevention and management of pain in neonates admitted to the NICU $(21,22)$. Simple pharmacological and non-pharmacological methods that reduce pain are not practiced. Previous studies show that NICU staff lack knowledge about neonatal pain, and that their attitudes hinder pain management in neonates $(22,23)$.

\section{Goals and Objectives}

The present study sought to determine the number of painful procedures performed per day for each neonate to verify the documentation of painful procedures performed and to, subsequently, note the number of missed opportunities for providing pain relief to neonates at an NICU in western India.

\section{MATERIALS AND METHODS}

\section{Setting}

We conducted a cross-sectional observational study at the level III NICU of Shri Krishna Hospital in Karamsad, which is a rural village located in the state of Gujarat, in western India.

\section{Definitions}

A procedure was defined as "a sporadic medical, nursing or surgical, and diagnostic or therapeutic activity performed on the child" (14). This definition implies that a continuous therapeutic procedure could not be counted as a procedure, e.g., ventilation. In the absence of an appropriate/accurate definition of neonatal pain, we chose to use the IASP definition of pain (1). A procedure was considered painful if it "invaded the neonate's bodily integrity, causing skin injury or mucosal injury from the introduction or removal of foreign material into the airway or the digestive or urinary tract" (14). A procedure was considered to be stressful when it "disturbed the equilibrium that was existent between the neonate and its environment or annoyed the neonate" (14). All of the procedures were classified as either stressful or mildly, moderately, or severely painful, as described by the NNF Clinical Practice Guidelines (13). A panel of four neonatologists reviewed the NNF procedural pain classification and tailored it to the present study. Handling by caregivers was considered stressful. Maternal touch was not deemed to be stressful.

\section{Subject Recruitment and Study Procedure}

The study was conducted in two phases. The first phase was an observational study of 29 neonates. The purpose of this phase was to determine the accuracy of documentation. Only neonates admitted for more than 1 day were included in this phase. The second phase comprised a retrospective assessment of the case records of 40 neonates. The first 7 days of NICU admission were reviewed from the neonatal case records. All of the documented stressful and painful procedures were recorded in a predesigned pro forma for each neonate. The same file audit method was used in both phases.

\section{First Phase}

In the first phase, one of the authors directly observed 29 neonates in the NICU for a total of 738 patient h over 17 days. The number of painful procedures documented in each neonate's respective case record was compared to the number of procedures directly observed. Pain relief measures were also recorded and compared to the documented data. 
Our NICU is so structured that an observer in the NICU can monitor activities in four to five neonates at a time. Twenty-nine infants were observed for $24 \mathrm{~h}$ each. The observation was divided into blocks of 4-6 h spread over 2-3 days. Once $24 \mathrm{~h}$ of observation per neonate was completed, that particular neonate was not included further for data collection. No fixed timings were determined for observation; time slots were chosen randomly. However, we ensured that all periods of the day and night were covered to minimize bias. Average nurse-patient ratio was also calculated, by averaging the ratios from each of the various time slots during which the neonates were observed.

The neonates' case records were then reviewed for documentation, and a documentation deficit was calculated based on the actual documentation of procedures by the investigators versus the entries done by the NICU staff in the patient records. The documentation check was done after the live data collection was completed, in the hospital medical records department.

\section{Second Phase}

Neonatal intensive care unit admissions data was retrieved from the hospital medical records department. Neonates having admission for more than 7 days were carved out from the data. Then, using the random number generation feature of Microsoft Excel, 40 files were selected for review.

Both nursing notes and resident/doctor notes, along with other documentation in the case records like investigation sheets and drug charts, were reviewed to determine the number of procedures performed and to determine the number of instances during which pharmaceutical pain relief agents, and/or central nervous system depressants (barbiturates, benzodiazepines), were administered before or at the time of the procedures. The specific drug administered, the drug's known duration of action, time of drug administration, and time and length of the procedure were all taken into consideration when determining whether a central nervous or nociceptive depressant action was in effect for the duration of each procedure. This was done during the first phase as well.

The documentation deficit from the first phase was used to calculate projected numbers of procedures occurring each day for these 40 neonates.

\section{Ethics Approval}

The institutional Human Research and Ethics Committee (HREC) approved the study and also duly approved a waiver of written informed consent.

\section{Data Analysis}

The data was entered into Microsoft Excel. It was then analyzed using a combination of Microsoft Excel and Stata 14, with descriptive statistics.

\section{RESULTS}

The study included 69 neonates, 29 of whom were directly observed in the NICU for a total of 738 patient h, and 40 of which whose patient records were retrospectively studied for the first 7 days of admission in the NICU. In the first phase, 2121 procedures were observed, of which 2075 were noted in their respective neonates' case records. There was, therefore, a deficit in documentation for $2.2 \%$ of the procedures. Considering this, the authors decided to ignore the minor deficit in documentation and decided to analyze all 69 of the patient records together. On direct observation of the 29 neonates, it was observed that the average patient-nurse ratio was 1.53:1 at the center where the present study was conducted.

A majority of the 69 neonates were male, preterm, born by Cesarean section, and had low birth weight and poor APGAR score at 1 min after birth. A large proportion developed early onset sepsis. Respiratory distress and hypoxic ischemic encephalopathy were also quite common. A large proportion required invasive or non-invasive airway support and long stay in the NICU. Nearly half of the neonates had abnormal neurology at the time of admission. See Table 1 for sociodemographic and clinical profile of the neonates, and Table 2 for common clinical diagnoses.

Overall, a total number of 13711 procedures were recorded. This amounted to 44.1 procedures per patient day. Of these 44.1 procedures per patient day, 38.1 were stressful procedures, 3.8 were mildly painful, and 2.2 were moderately painful procedures. Please see Table 3 for the specifics of each phase.

Table 4 depicts the common procedures observed and documented. The most common stressful, mildly painful, and moderately painful procedures were position-changing, heel prick for blood sugar testing, and endotracheal suctioning, respectively. Severely painful procedures entail surgical procedures, which were not encountered in any of these neonates.

A majority of painful procedures occurred over the first few days of admission. There was a general trend of decreasing numbers of painful procedures as the duration of admission progressed. Such a trend has not been observed for the stressful procedures (Figure 1).

A total of 27 (39.1\%) out of 69 neonates received some pharmaceutical agent for pain relief/sedation/central nervous system depression. Such pharmaceutical agents were used for 4592 $(33.5 \%)$ of the procedures. Lorazepam $(1169 ; 25.5 \%)$, phenobarbitone $(1145 ; 24.9 \%)$, ibuprofen $(741 ; 16.1 \%)$, midazolam (568; $12.4 \%)$, fentanyl $(495 ; 10.8 \%)$ and morphine $(242 ; 5.3 \%)$ were the commonly used pharmacological agents (Figure 2). The use of pharmacological agents did not correlate with the incidence of painful procedures.

\section{DISCUSSION}

A total of 44.1 procedures were recorded per patient day. There were 3.8 mildly painful procedures and 2.2 moderately painful procedures per patient day; these are significant numbers and imply that the neonates undergo a very high level of daily stress, which can have adverse effects on their health and neurodevelopment in the near future (Table 2). The first 7 days of admission were reviewed because the highest number of NICU interventions occurs for neonates weighing $>1000 \mathrm{~g}$, which comprise a large proportion of our admissions. An important observation was that there was a decrease in the incidence of painful procedures as 
TABLE 1 | Sociodemographic and clinical profile of neonates.

\begin{tabular}{|c|c|c|c|c|c|c|c|c|c|}
\hline & \multicolumn{3}{|c|}{29 observed neonates } & \multicolumn{3}{|c|}{40 file audits } & \multicolumn{3}{|c|}{ All 69 neonates } \\
\hline & Pain relief & Non-pain relief & Total & Pain relief & Non-pain relief & Total & Pain relief & Non-pain relief & Total \\
\hline \multicolumn{10}{|l|}{ Sex } \\
\hline Male & 9 & 10 & 19 & 12 & 15 & 27 & 21 & 25 & 46 \\
\hline Female & 3 & 7 & 10 & 3 & 10 & 13 & 6 & 17 & 23 \\
\hline \multicolumn{10}{|l|}{ Place of birth } \\
\hline Outborn & 7 & 9 & 16 & 6 & 6 & 12 & 13 & 15 & 28 \\
\hline Inborn & 5 & 8 & 13 & 9 & 19 & 28 & 14 & 27 & 41 \\
\hline \multicolumn{10}{|l|}{ Birth weight (kg) } \\
\hline Mean (SD) & $\begin{array}{c}2.062 \\
(0.8377)\end{array}$ & $\begin{array}{c}1.646 \\
(0.6438)\end{array}$ & $\begin{array}{c}1.818 \\
(0.7457)\end{array}$ & $\begin{array}{c}2.222 \\
(0.5427)\end{array}$ & $\begin{array}{c}1.924 \\
(0.5311)\end{array}$ & $\begin{array}{c}2.036 \\
(0.5480)\end{array}$ & $\begin{array}{c}2.151 \\
(0.6798)\end{array}$ & $\begin{array}{c}1.812 \\
(0.5883)\end{array}$ & $\begin{array}{c}1.944 \\
(0.6428)\end{array}$ \\
\hline Normal ( $\geq 2.5$ kg) & 5 & 3 & 8 & 6 & 5 & 11 & 11 & 8 & 19 \\
\hline LBW $(<2.5 \mathrm{~kg})$ & 3 & 8 & 11 & 7 & 16 & 23 & 10 & 24 & 34 \\
\hline VLBW (<1.5 kg) & 3 & 4 & 7 & 2 & 4 & 6 & 5 & 8 & 13 \\
\hline ELBW $(<1 \mathrm{~kg})$ & 1 & 2 & 3 & 0 & 0 & 0 & 1 & 2 & 3 \\
\hline \multicolumn{10}{|l|}{ Gestational age (weeks) } \\
\hline Mean (SD) & $35.5(4.10)$ & 34.5 (3.99) & 34.9 (3.99) & 36.3 (2.99) & $35.5(2.63)$ & $35.8(3.76)$ & $35.9(3.47)$ & $35.1(3.24)$ & $35.4(3.33)$ \\
\hline \multicolumn{10}{|l|}{ Full term } \\
\hline ( $\geq 37$ weeks) & 6 & 6 & 12 & 6 & 7 & 13 & 12 & 13 & 25 \\
\hline \multicolumn{10}{|l|}{ Late preterm } \\
\hline (34-36 weeks) & 2 & 3 & 5 & 6 & 14 & 20 & 8 & 17 & 25 \\
\hline \multicolumn{10}{|l|}{ Early preterm } \\
\hline (32-34 weeks) & 1 & 3 & 4 & 2 & 2 & 4 & 3 & 5 & 8 \\
\hline (30-32 weeks) & 1 & 3 & 4 & 1 & 1 & 2 & 2 & 4 & 6 \\
\hline (28-30 weeks) & 2 & 1 & 3 & 0 & 1 & 1 & 2 & 2 & 4 \\
\hline (26-28 weeks) & 0 & 1 & 1 & 0 & 0 & 0 & 0 & 1 & 1 \\
\hline Gestational maturity & & & & & & & & & \\
\hline AGA & 8 & 5 & 13 & 8 & 14 & 22 & 16 & 19 & 35 \\
\hline SGA & 4 & 12 & 16 & 7 & 11 & 18 & 11 & 23 & 34 \\
\hline Head circumference & & & & & & & & & \\
\hline Mean (SD) & $31.2(4.05)$ & $28.8(3.26)$ & $29.8(3.75)$ & 31.7 (2.03) & $30.4(2.65)$ & $30.9(2.49)$ & $31.5(3.04)$ & $29.8(2.99)$ & $30.4(3.11)$ \\
\hline Length & & & & & & & & & \\
\hline Mean (SD) & $45.4(4.64)$ & $42.0(4.70)$ & $43.4(4.90)$ & $45.4(3.58)$ & $43.3(4.61)$ & $44.2(4.33)$ & $45.4(4.00)$ & $42.8(4.64)$ & $43.8(4.56)$ \\
\hline Mode of birth & & & & & & & & & \\
\hline Spontaneous vaginal delivery & 6 & 11 & 17 & 5 & 6 & 11 & 11 & 17 & 28 \\
\hline LSCS under spinal anesthesia & 5 & 5 & 10 & 6 & 12 & 18 & 11 & 17 & 28 \\
\hline LSCS under general anesthesia & 0 & 1 & 1 & 2 & 7 & 9 & 2 & 8 & 10 \\
\hline Assisted vaginal delivery (vacuum) & 1 & 0 & 1 & 0 & 0 & 0 & 1 & 0 & 1 \\
\hline Assisted vaginal delivery (forceps) & 0 & 0 & 0 & 2 & 0 & 2 & 2 & 0 & 2 \\
\hline Liquor & & & & & & & & & \\
\hline Clear & 4 & 7 & 11 & 7 & 14 & 21 & 11 & 21 & 32 \\
\hline Meconium stained & 2 & 2 & 4 & 5 & 7 & 12 & 7 & 9 & 16 \\
\hline Not known & 6 & 8 & 14 & 3 & 4 & 7 & 9 & 12 & 21 \\
\hline Cried immediately after birth & & & & & & & & & \\
\hline Cried immediately & 5 & 12 & 17 & 5 & 7 & 12 & 10 & 19 & 29 \\
\hline Weak cry & 1 & 2 & 3 & 3 & 5 & 8 & 4 & 7 & 11 \\
\hline Did not cry immediately & 6 & 3 & 9 & 7 & 13 & 20 & 13 & 16 & 29 \\
\hline Bag/mask required & & & & & & & & & \\
\hline Yes & 8 & 4 & 12 & 11 & 16 & 27 & 19 & 20 & 39 \\
\hline No & 4 & 13 & 17 & 4 & 9 & 13 & 8 & 22 & 30 \\
\hline APGAR score & & & & & & & & & \\
\hline After 1 min [mean (SD)] & $4.4(1.52)$ & $5.5(1.93)$ & $5.08(1.80)$ & $4.6(1.94)$ & $4.62(2.22)$ & $4.60(2.11)$ & $4.5(1.74)$ & $4.86(2.15)$ & $4.74(2.01)$ \\
\hline After 5 min [mean (SD)] & $6.4(2.07)$ & $8.13(1.36)$ & $7.46(1.81)$ & $7.0(2.35)$ & $7.24(1.67)$ & $7.17(1.86)$ & $6.8(2.2)$ & $7.48(1.62)$ & $7.26(1.83)$ \\
\hline After 10 min [mean (SD)] & $6.0(1.41)$ & $8.00(0.0)$ & $7.00(1.41)$ & $7.3(2.31)$ & $7.71(1.38)$ & $7.60(1.58)$ & $6.8(1.9)$ & $7.78(1.20)$ & $7.43(1.50)$ \\
\hline Maternal high risk factors prese & & & & & & & & & \\
\hline Yes & 9 & 15 & 24 & 14 & 23 & 37 & 23 & 38 & 61 \\
\hline No & 3 & 2 & 5 & 1 & 2 & 3 & 4 & 4 & 8 \\
\hline Neurological status & & & & & & & & & \\
\hline Good & 2 & 11 & 13 & 8 & 17 & 25 & 10 & 28 & 38 \\
\hline Poor & 10 & 6 & 16 & 7 & 8 & 15 & 17 & 14 & 31 \\
\hline Age on admission (number of de & & & & & & & & & \\
\hline Mean (SD) & $1.42(0.793)$ & $1.53(1.23)$ & $1.48(1.06)$ & $1.40(1.056)$ & $2.56(5.66)$ & $2.13(4.52)$ & $1.41(0.931)$ & $2.14(4.43)$ & $1.86(3.50)$ \\
\hline Age on discharge (number of da & & & & & & & & & \\
\hline Mean (SD) & $14.4(17.1)$ & $11.0(9.25)$ & $12.4(12.9)$ & $15.1(9.82)$ & $15.0(9.13)$ & $15.1(9.27)$ & $14.8(13.3)$ & $13.4(9.28)$ & $14.0(10.9)$ \\
\hline Duration of stay (days) & & & & & & & & & \\
\hline Mean (SD) & $14.0(17.2)$ & $10.5(9.18)$ & $11.9(13.0)$ & $14.8(9.92)$ & $13.5(7.82)$ & $14.0(8.56)$ & $14.4(13.4)$ & $12.3(8.42)$ & $13.1(10.6)$ \\
\hline Nil by mouth & & & & & & & & & \\
\hline Yes & 9 & 3 & 12 & 8 & 4 & 12 & 17 & 7 & 24 \\
\hline No & 3 & 14 & 17 & 7 & 21 & 28 & 10 & 35 & 45 \\
\hline
\end{tabular}

"Pain relief" neonates are those receiving pharmaceutical agents for at least one procedure. "Non-pain relief" neonates have not received any pharmaceutical pain relief/central nervous system depressing agents. LSCS, lower segment Cesarean section. 
TABLE 2 | Common clinical diagnoses.

\begin{tabular}{ll}
\hline $\mathbf{2 9}$ observed patients (frequency) & \multicolumn{1}{c}{$\mathbf{4 0}$ file audits (frequency) } \\
\hline Early onset sepsis (21) & Early onset sepsis (21) \\
Respiratory distress syndrome (12) & Respiratory distress syndrome (14) \\
Hyperbilirubinemia (7) & Meconium stained liquor (11) \\
Apnea of prematurity (5) & On ventilatory support (11) \\
On ventilatory support (5) & Birth asphyxia (8) \\
\hline
\end{tabular}

TABLE 3 | Procedure documentation data.

\begin{tabular}{|c|c|c|c|c|}
\hline & \multicolumn{2}{|c|}{$\begin{array}{l}\text { Observed patients and } \\
\text { files (29) }\end{array}$} & \multicolumn{2}{|c|}{ File audits (40) } \\
\hline & Observed & Documented & Documented & Projected \\
\hline $\begin{array}{l}\text { Total number of } \\
\text { procedures }\end{array}$ & 2121 & 2075 & 11636 & 11892 \\
\hline $\begin{array}{l}\text { Procedures per } \\
\text { patient } \mathrm{h}\end{array}$ & 2.87 & 2.81 & 1.73 & 1.77 \\
\hline $\begin{array}{l}\text { Stressful } \\
\text { procedures }\end{array}$ & 1705 & 1691 & 10170 & 10394 \\
\hline $\begin{array}{l}\text { Stressful } \\
\text { procedures/ } \\
\text { patient h }\end{array}$ & 2.31 & 2.29 & 1.51 & 1.52 \\
\hline $\begin{array}{l}\text { Mildly painful } \\
\text { procedures }\end{array}$ & 219 & 195 & 985 & 1007 \\
\hline $\begin{array}{l}\text { Mildly painful } \\
\text { procedures/ } \\
\text { patient h }\end{array}$ & 0.3 & 0.26 & 0.15 & 0.17 \\
\hline $\begin{array}{l}\text { Moderately painful } \\
\text { procedures }\end{array}$ & 197 & 189 & 481 & 492 \\
\hline $\begin{array}{l}\text { Moderately painful } \\
\text { procedures/ } \\
\text { patient h }\end{array}$ & 0.27 & 0.26 & 0.07 & 0.07 \\
\hline
\end{tabular}

TABLE 4 | Common procedures.

\begin{tabular}{lll}
\hline & \multicolumn{1}{c}{ 29 observed patients } & \multicolumn{1}{c}{ 40 file audits } \\
Most & Position change (311) & Position Change (2190) \\
common & Temperature (283) & Temperature (1925) \\
stressful & Abdominal girth (280) & Abdominal girth (1888) \\
procedures & Diaper change (280) & Diaper change (1795) \\
(frequency) & Physical exam (278) & Physical exam (1340) \\
Most & Heel prick (130) & Heel prick (627) \\
common & Venipuncture (27) & Blood sample (143) \\
mildly painful & Blood sample (18) & Venipuncture (62) \\
procedures & NT insertion (14) & Umbilical catheterization (62) \\
(frequency) & Umbilical catheterization (7) & IV removal (25) \\
Most & Suction (178) & Suction (351) \\
common & Lumbar puncture (5) & Remove ET (19) \\
moderately & Change umbilical dressing (4) & Change umbilical dressing (23) \\
painful & ET adjust (3) & ET insertion (30) \\
procedures & Remove umbilical dressing (2) & Remove umbilical dressing (37) \\
(frequency) & &
\end{tabular}

NT, nasogastric tube; ET, endotracheal tube.

the stay of the neonate progressed (Figure 1). This reflects upon the fact that neonates require most procedures on the first day of admission and that they tend to stabilize subsequently and therefore require less painful interventions. It also reflects the seriousness and subsequent gradual improvement of the neonates' general condition and suggests that this is the peak time for implementing effective pain relief measures to ensure adequate pain management.

Other studies have also recorded a similarly high number of stressful/painful procedures in the $\operatorname{NICU}(14,22)$. We found position changing, abdominal girth measurement, temperature recording, diaper changing, and physical examination were the most common stressful procedures (Table 3). This is similar to other studies $(21,22)$. Such stressful/painful procedures always occur as a part of neonatal intensive care and are inevitable (21). Clubbing of these procedures is a routine practice at the study center. Minimizing unnecessary procedures, and practicing evidence-based medicine, are the key to easing pain in these neonates.

Non-pharmacological measures of pain relief have been proven effective for pain relief during stressful and mildly to moderately painful procedures $(13,24-32)$. Kangaroo mother care, clubbing of procedures at time of breast milk feeding, nonnutritive sucking, swaddling, and oral sweet tasting solutions are regular practices at the center.

Pharmacological measures of pain relief, such as non-steroidal anti-inflammatory drugs (NSAIDs) or opioids/anti-convulsants/ sedatives/other central nervous system depressants that partially or completely depress nociceptive pathways, for such short term and mild to moderate procedural pain, are not recommended on account of their poor efficacy and the encountered side effects (33-35). Such measures were used only one-third of the time. The drugs used were inappropriate and, as we believe, were at many times not intended for pain relief. They were instead administered either as sedation for ventilation or were intended for another underlying pathology altogether, such as convulsions or congenital heart disease. As observed in Figure 2, the frequency of pharmacological agent distribution does not correlate with the frequency of painful procedures. This also indirectly indicates that the pharmacological agents were not intended for pain relief. A majority of neonates on ventilator support were only administered barbiturates like phenobarbitone, with no supplementary analgesics. Barbiturates do not provide analgesia; rather, they only provide sedation, anxiolysis, muscle relaxation and amnesia and may even suppress clinical signs of neonatal pain (36). These neonates were therefore under a high amount of unnoticed pain/stress, leading to possible neurological insult with future repercussions in childhood.

Thirty-one patients (44.9\%) were neurologically abnormal at the time of admission. Nursing staff may be misled into believing that absence of response in these neonates implies absence of pain. More than $50 \%$ of the neonates were born by Cesarean section. Studies demonstrate a dampened pain response in neonates during and up to several hours after vaginal delivery due to catecholamine surge and sympathoadrenal activation, which is absent in neonates born by Cesarean section (37). The importance of this is that neonates born by Cesarean section have a lower threshold for pain than vaginally born neonates.

The nursing staff in the present study documented a majority of the invasive and non-invasive procedures. This is a reassuring 


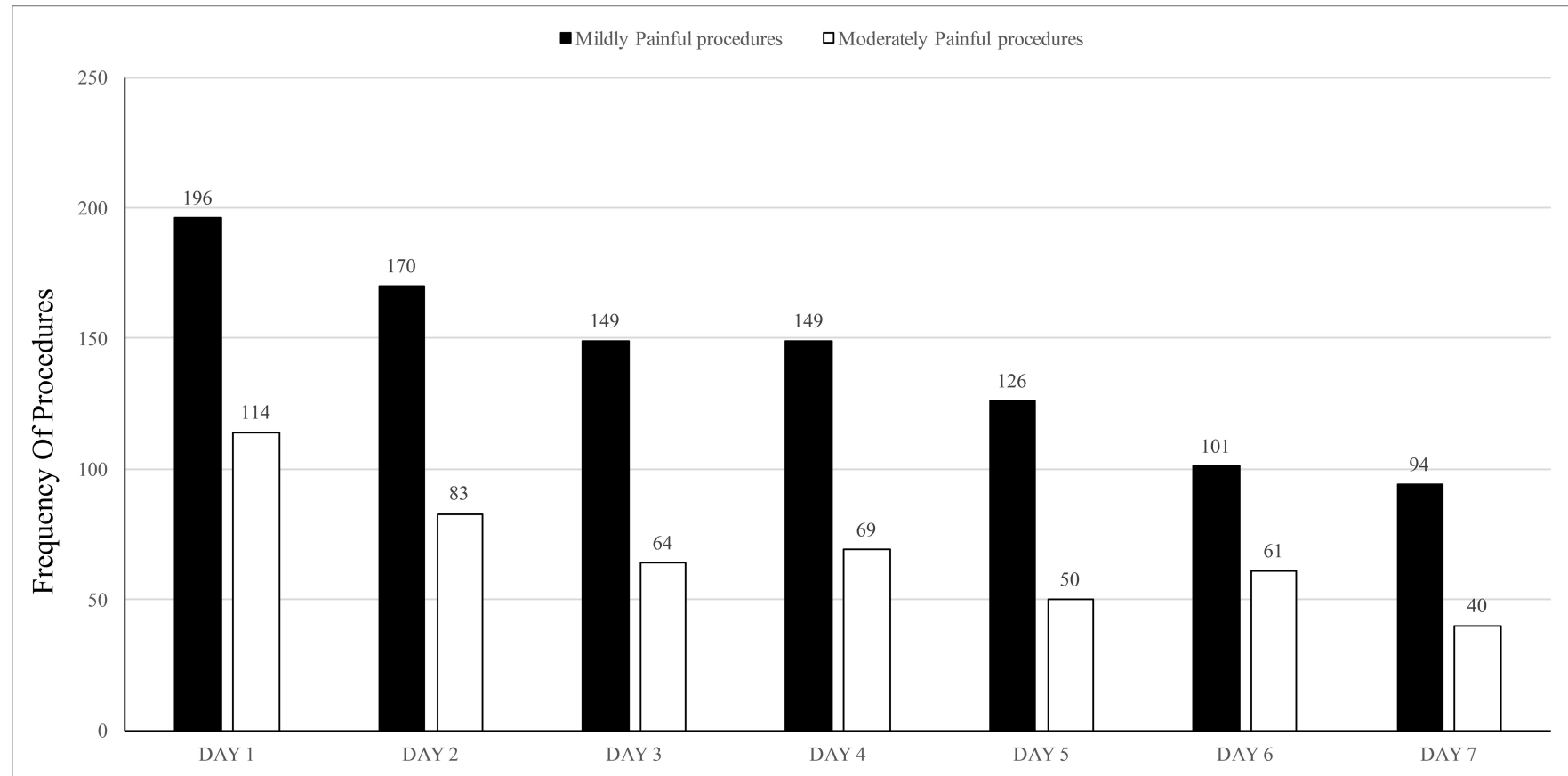

FIGURE 1 | Distribution of painful procedures over the first 7 days of admission.

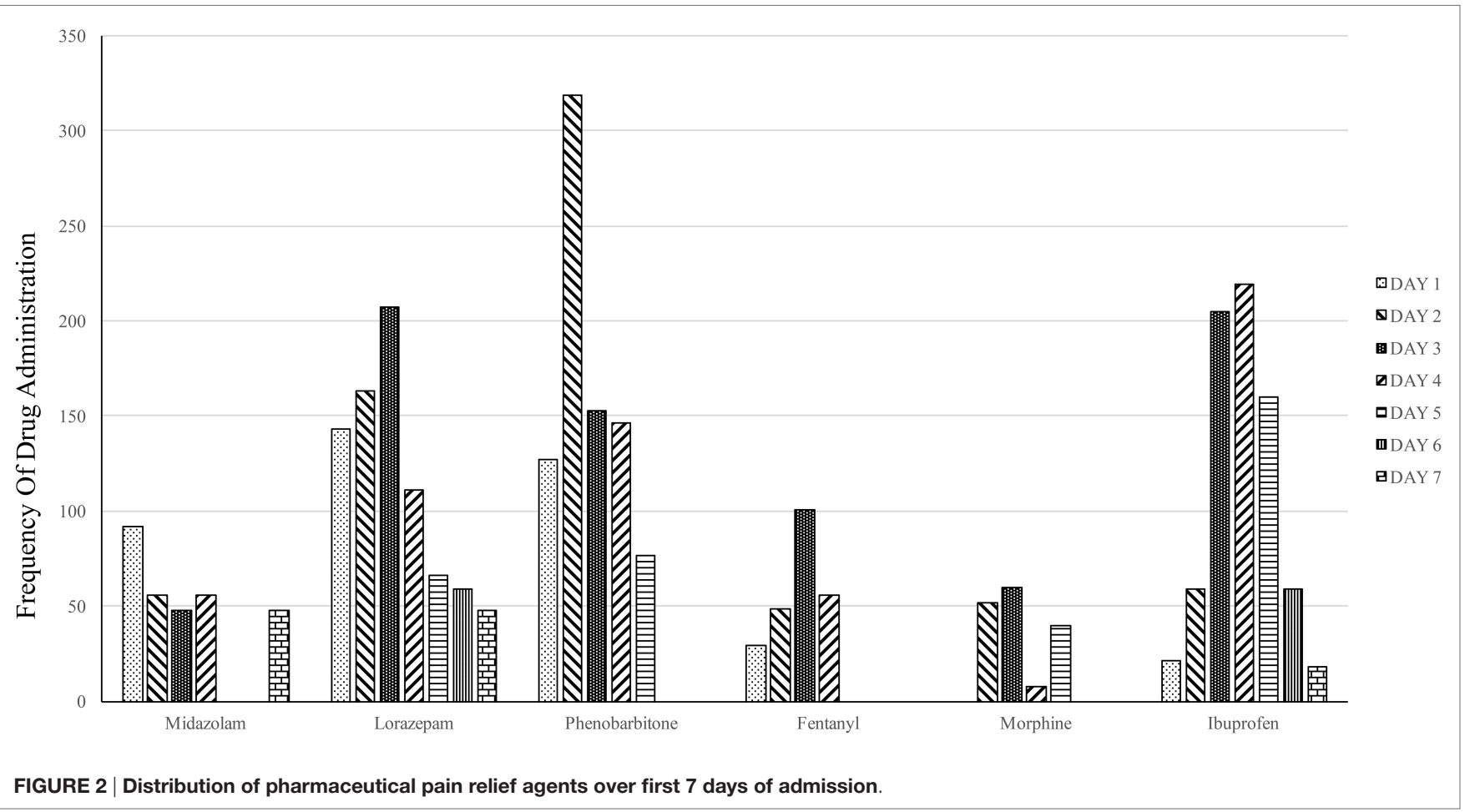

fact, indirectly indicating that the staff was sensitive even toward stressful procedures. The nurse-patient ratio at the study center is better than most centers in India (38). Therefore, the authors are of the opinion that this finding regarding documentation practices cannot be generalized to all NICU's in India, as most of them are not as well staffed (39).
Previous studies conducted at the present institute reflected upon the poor knowledge and attitudes of nurses toward pain $(40,41)$. The present study reflects on their poor pain management practices. This not only depicts the developing country scenario; it epitomizes the developed country scenario as well (42-44). 
Our study design had inherent limitations. The directly observed study of 29 patients was not blinded. Nurses were only informed that the number of procedures was being observed; they were not informed that their documentation would be assessed. Even the mere presence of an observer can result in a falsely improved performance; therefore, it can falsely depict increased documentation. The second part of the study depends on retrospective assessment of records. General timings of drug administration and timings of procedures were documented and, accordingly, correlated; however, it was not feasible to document the exact time of occurrence of the procedure and correlate it with the exact duration of the administered drug's effect. We also could not document the indication for the medication administration. Use of non-pharmacological methods of pain relief was not documented and therefore not included in the observations. Limitations notwithstanding the study throws up important findings, which, if addressed, can improve the quality of neonatal care.

Interventions targeting nurses' knowledge, attitudes, and practices regarding neonatal pain need to be undertaken. Doctors and residents, too, should have increased interest in neonatal pain, as without them it is nearly impossible to create a minimal pain environment for neonates (45). Studies conducted previously state that the identification of missed opportunities and utilization of appropriate interventions and implementation of treatment protocols can improve health care substantially (46).

\section{CONCLUSION}

Neonatal pain is a fairly common entity in the NICU, and appropriate identification and management is a necessity. Stressful procedures are very common in the NICU, and mildly and moderately painful procedures are fairly common. Proper and adequate pain relief measures are seldom used. There is an urgent need for sensitization and education of all personnel involved in neonatal care. Nurses, being the primary caregivers, are the mainstay for addressing this issue; therefore, neonatal pain should be incorporated into routine nursing curriculum. Improved education of the doctors and residents caring for neonates is also of utmost importance, so that unnecessary painful interventions are avoided and proper prevention and treatment protocols for

\section{REFERENCES}

1. IASP Taxonomy. International Association of Study of Pain (2011). Available from: http://www.iasp-pain.org

2. Anand KJ, Craig KD. New perspectives on the definition of pain. Pain (1996) 67(1):3-6.

3. Vani S, Thakre R, Nimbalkar S. Research issues in assessment and management of pain in newborns. J Neonatol (2009) 24(3):358-63.

4. Anderson RD, Greve-Isdahl M, Jylli L. The opinions of clinical staff regarding neonatal procedural pain in two Norwegian neonatal intensive care units. Acta Paediatr (2007) 96:1000-3. doi:10.1111/j.1651-2227.2007.00190.x

5. Anand KJ. Pain assessment in preterm neonates. Pediatrics (2007) 119(3):605-7. doi:10.1542/peds.2006-2723

6. Mountcastle K. An ounce of prevention: decreasing painful interventions in the NICU. Neonatal Netw (2010) 29(6):353-8. doi:10.1891/0730-0832.29.6.353 neonatal pain can be established, thereby creating a minimal pain environment for neonates.

\section{AUTHOR CONTRIBUTIONS}

SK contributed to the design of the study, data acquisition, data analysis, revision of the manuscript for important intellectual content, and final approval of this manuscript. AD contributed to the design of the study, data analysis, writing the manuscript, and final approval of the manuscript. SN contributed to the design and planning of the study, data analysis, revision of the manuscript for important intellectual content, and final approval of this manuscript. AP contributed to study design, data analysis, and revision of the manuscript for important intellectual content and final approval of manuscript. AN contributed to data acquisition, revision of the manuscript for important intellectual content, and final approval of manuscript. All authors agree to be accountable to all aspects of the work, and SN will be the guarantor for the paper.

\section{ACKNOWLEDGMENTS}

The authors sincerely acknowledge the Medical Records Department at Shri Krishna Hospital for providing the patient files for both phases of the study. The authors also acknowledge Mr. Binoy Shah for creating high quality versions of Figures 1 and 2.

\section{FUNDING}

SK received funding under the 2013 Short Term Studentship (STS) program by the Indian Council of Medical Research (ICMR) (Ref. No. 2013-00036). ICMR STS awarded Rs. 10,000 (\$167) to SK for this study. The ICMR also awarded SK "International Travel Grant for Non-I CMR Young Scientists.” This covered the costs of traveling to Vancouver, Canada for presenting the abstract at the Pediatric Academic Societies/Asian Society for Pediatric Research (PAS/ASPR) Annual Meeting, 2014 (held in Vancouver, Canada).

\section{SUPPLEMENTARY MATERIAL}

The Supplementary Material for this article can be found online at http://journal.frontiersin.org/article/10.3389/fped.2016.00007

7. Walker SM. Management of procedural pain in NICUs remains problematic. Paediatr Anaesth (2005) 15:909-12. doi:10.1111/j.1460-9592.2005.01758.x

8. Taddio A, Shah V, Gilbert-MacLeod C, Katz J. Conditioning and hyperalgesia in newborns exposed to repeated heel lances. JAMA (2002) 288(7):857-61. doi:10.1001/jama.288.7.857

9. Helmuth L. Neuroscience. Early insult rewires pain circuits. Science (2000) 289(5479):521-2. doi:10.1126/science.289.5479.521a

10. Grunau R. Early pain in preterm infants. A model of long-term effects. Clin Perinatol (2002) 29(3):373-94, vii-viii. doi:10.1016/ S0095-5108(02)00012-X

11. Lidow MS. Long-term effects of neonatal pain on nociceptive systems. Pain (2002) 99(3):377-83. doi:10.1016/S0304-3959(02)00258-0

12. Brummelte S, Grunau RE, Chau V, Poskitt KJ, Brant R, Vinall J, et al. Procedural pain and brain development in premature newborns. Ann Neurol (2012) 71(3):385-96. doi:10.1002/ana.22267 
13. Vani SN, Thakre R, Nimbalkar S. Assessment and management of pain in the newborn. NNF Clin Pract Guidel (2010):199-215.

14. Carbajal R, Rousset A, Danan C, Coquery S, Nolent P, Ducrocq S, et al. Epidemiology and treatment of painful procedures in neonates in intensive care units. JAMA (2008) 300(1):60-70. doi:10.1001/jama.300.1.60

15. Walter-Nicolet E, Annequin D, Biran V, Mitanchez D, Tourniaire B. Pain management in newborns: from prevention to treatment. Paediatr Drugs (2010) 12(6):353-65. doi:10.2165/11318900-000000000-00000

16. Cignacco E, Hamers J, van Lingen RA, Stoffel L, Buchi S, Muller R, et al. Neonatal procedural pain exposure and pain management in ventilated preterm infants during the first 14 days of life. Swiss Med Wkly (2009) 139(15-16):226-32.

17. Anand KJ; International Evidence-Based Group for Neonatal Pain. Consensus statement for the prevention and management of pain in the newborn. Arch Pediatr Adolesc Med (2001) 155(2):173. doi:10.1001/archpedi.155.2.173

18. Anand KJ, Hall RW. Pharmacological therapy for analgesia and sedation in the newborn. Arch Dis Child Fetal Neonatal Ed (2006) 91(6):F448. doi:10.1136/ adc. 2005.082263

19. Lago P, Garetti E, Merazzi D, Pieragostini L, Ancora G, Pirelli A, et al. Guidelines for procedural pain in the newborn. Acta Paediatr (2009) 98(6):932. doi:10.1111/j.1651-2227.2009.01291.x

20. American Academy of Pediatrics Committee on Fetus and Newborn; American Academy of Pediatrics Section on Surgery; Canadian Paediatric Society Fetus and Newborn Committee; Batton DG, Barrington KJ, Wallman C. Prevention and management of neonatal pain: an update. Pediatrics (2006) 118(5):2231. doi:10.1542/peds.2006-2277

21. Nimbalkar AS, Dongara AR, Ganjiwale JD, Nimbalkar SM. Pain in children: knowledge and perceptions of the nursing staff at a rural tertiary care teaching hospital in India. Indian J Pediatr (2013) 80(6):470-5. doi:10.1007/s12098-012-0848-z

22. Nimbalkar AS, Dongara AR, Phatak AG, Nimbalkar SM. Knowledge and attitudes regarding neonatal pain among nursing staff of pediatric department: an Indian experience. Pain Manag Nurs (2014) 15(1):69-75. doi:10.1016/j.pmn.2012.06.005

23. Kalyan G, Vatsa $M$. Neonatal nursing: an unmet challenge in India. Indian J Pediatr (2014) 81:1205-11. doi:10.1007/s12098-014-1567-4

24. Nimbalkar S, Sinojia A, Dongara A. Reduction of neonatal pain following administration of $25 \%$ lingual dextrose: a randomized control trial. J Trop Pediatr (2013) 59(3):223-5. doi:10.1093/tropej/fms072

25. Nimbalkar SM, Chaudhary NS, Gadhavi KV, Phatak A. Kangaroo mother care in reducing pain in preterm neonates on heel prick. Indian J Pediatr (2013) 80(1):6-10. doi:10.1007/s12098-012-0760-6

26. Barker DP, Rutter N. Exposure to invasive procedures in neonatal intensive care unit admissions. Arch Dis Child Fetal Neonatal Ed (1995) 72(1):F47-8. doi:10.1136/fn.72.1.F47

27. Bellieni CV, Cordelli DM, Marchi S, Ceccarelli S, Perrone S, Maffei M, et al. Sensorial saturation for neonatal analgesia. Clin J Pain (2007) 23(3):219. doi:10.1097/AJP.0b013e31802e3bd7

28. Golianu B, Krane E, Seybold J, Almgren C, Anand KJ. Non pharmacological techniques for pain management in neonates. Semin Perinatol (2007) 31(5):318. doi:10.1053/j.semperi.2007.07.007

29. Chermont AG, Falcão LF, de Souza Silva EH, de Cássia Xavier Balda R, Guinsburg R. Skin-to-skin contact and/or oral 25\% dextrose for procedural pain relief for term newborn infants. Pediatrics (2009) 124(6):e1101. doi:10.1542/peds.2009-0993

30. Marín Gabriel ḾA, del Rey Hurtado de Mendoza B, Jiménez Figueroa L, Medina V, Iglesias Fernández B, Vázquez Rodríguez M, et al. Analgesia with breastfeeding in addition to skin-to-skin contact during heel prick. Arch Dis Child Fetal Neonatal Ed (2013) 908(6):F499. doi:10.1136/ archdischild-2012-302921
31. Okan F, Coban A, Ince Z, Yapici Z, Can G. Analgesia in preterm newborns: the comparative effects of sucrose and glucose. Eur J Pediatr (2007) 166(10):1017. doi:10.1007/s00431-006-0373-z

32. Pillai Riddell RR, Racine NM, Turcotte K, Uman LS, Horton RE, Din Osmun L, et al. Non-pharmacological management of infant and young child procedural pain. Cochrane Database Syst Rev (2011) 10:CD006275. doi:10.1002/14651858.CD006275.pub3

33. Stevens B, Yamada J, Lee GY, Ohlsson A. Sucrose for analgesia in newborn infants undergoing painful procedures. Cochrane Database Syst Rev (2013) 1:CD001069. doi:10.1002/14651858.CD001069.pub4

34. Shah PS, Herbozo C, Aliwalas LL, Shah VS. Breastfeeding or breast milk for procedural pain in neonates. Cochrane Database Syst Rev (2012) 12:CD004950. doi:10.1002/14651858.CD004950.pub3

35. Bhatt-Mehta V, Rosen DA. Management of acute pain in children. Clin Pharm (1991) 10(9):667.

36. Anand KJS. Prevention and treatment of neonatal pain. In: Martin R, editor. UpToDate. Waltham, MA (2015). Available from: http://www.uptodate.com/ contents/prevention-and-treatment-of-neonatal-pain

37. Bergqvist LL, Katz-Salamon M, Hertegård S, Anand KJ, Lagercrantz H. Mode of delivery modulates physiological and behavioral responses to neonatal pain. J Perinatol (2009) 29(1):44-50. doi:10.1038/jp.2008.129

38. Fernandez A, Mondkar JA. Status of neonatal intensive care units in India. J Postgrad Med (1993) 39(2):57-9.

39. Amin AA, Vankar JR, Nimbalkar SM, Phatak AG. Perceived stress and quality of life in neonatal intensive care unit nurses in Gujarat, India. Indian J Pediatr (2015) 82(11):1001-5. doi:10.1007/s12098-015-1794-3

40. Shah V, Taddio A, Ohlsson A. Randomised controlled trial of paracetamol for heel prick pain in neonates. Arch Dis Child Fetal Neonatal Ed (1998) 79(3):F209-11. doi:10.1136/fn.79.3.F209

41. Carbajal R, Lenclen R, Jugie M, Paupe A, Barton BA, Anand KJ. Morphine does not provide adequate analgesia for acute procedural pain among preterm neonates. Pediatrics (2005) 115:1494-500. doi:10.1542/peds.2004-1425

42. Polkki T, Korhonen A, Laukkala H, Saarela T, Vehvilainen-Julkunen K, Pietila AM. Nurses' attitudes and perceptions of pain assessment in neonatal intensive care. Scand JCaring Sci (2010) 24(1):49-55. doi:10.1111/j.1471-6712.2008.00683.x

43. Pigeon HM, McGrath PJ, Lawrence J, MacMurray SB. Nurses' perceptions of pain in the neonatal intensive care unit. J Pain Symptom Manage (1989) 4(4):179-83. doi:10.1016/0885-3924(89)90040-7

44. Schollin J. Pain management in French neonatal intensive care units. Acta Paediatr (2002) 91(7):735-6. doi:10.1111/j.1651-2227.2002.tb03320.x

45. Lundeberg S. Pain in children - are we accomplishing the optimal pain treatment? Paediatr Anaesth (2015) 25(1):83-92. doi:10.1111/pan.12539

46. Hutchins SS, Jansen HA, Robertson SE, Evans P, Kim-Farley RJ. Studies of missed opportunities for immunization in developing and industrialized countries. Bull World Health Organ (1993) 71(5):549-60.

Conflict of Interest Statement: The authors declare that the research was conducted in the absence of any commercial or financial relationships that could be construed as a potential conflict of interest.

Copyright $\odot 2016$ Kothari, Dongara, Nimbalkar, Phatak and Nimbalkar. This is an open-access article distributed under the terms of the Creative Commons Attribution License (CC BY). The use, distribution or reproduction in other forums is permitted, provided the original author(s) or licensor are credited and that the original publication in this journal is cited, in accordance with accepted academic practice. No use, distribution or reproduction is permitted which does not comply with these terms. 